(2) Open Access Full Text Article

\title{
Stevens-Johnson Syndrome and toxic epidermal
} necrolysis: a multi-aspect comparative 7-year study from the People's Republic of China

This article was published in the following Dove Press journal:

Drug Design, Development and Therapy

12 December 2014

Number of times this article has been viewed

\author{
Jie Sun' \\ Jin Liu ${ }^{2}$ \\ Qing-Li Gong' \\ Gao-Zhong Ding' \\ Li-Wen Ma' \\ Li-Chao Zhang' \\ Yan Lu'
}

'Department of Dermatology, First Affiliated Hospital of Nanjing Medical University, ${ }^{2}$ Department of Epidemiology and Biostatistics, School of Public Health, Nanjing Medical University, Nanjing, Jiangsu Province, People's Republic of China
Correspondence: Yan Lu Department of Dermatology, First Affiliated Hospital of Nanjing Medical University, \#300 Guangzhou Road, Nanjing 210029, Jiangsu Province, People's Republic of China Tel +86 I39 I39 67II26

Fax $+86025837 \quad 8836$ Email luyan6289@I63.com
Background: Stevens-Johnson Syndrome (SJS) and toxic epidermal necrolysis (TEN) are rare but severe cutaneous drug reactions. They are differentiated based on the fraction of the body surface area affected. Optimal therapy for SJS and TEN is a controversial issue.

Objective: We compared the treatments given to and the clinical outcomes of 39 cases of SJS and 48 cases of TEN seen at a single institution between January 2007 and December 2013 for better understanding of the clinical characteristics and development of the two conditions.

Methods: Demographic data, clinical characteristics, treatments given, and therapeutic responses observed were retrospectively collected.

Results: The incidence rates of hypoproteinemia and secondary infections are significantly higher in TEN than in SJS ( $P=0.001$ and $P=0.002$, respectively). The corticosteroid dose did not influence the time from the initiation of therapy to control of the lesions in SJS, but increasing the dosage of corticosteroids progressively decreased the time from the initiation of therapy to control of the lesions in TEN. With increases in the utilization ratio of intravenous immunoglobulin (IVIG), the length of the hospital stay became shorter, whereas the time from the initiation of therapy to control of the lesions remained the same in SJS. However, for TEN, both the length of the hospital stay and the time from the initiation of therapy to control of the lesions became shorter with increases in the utilization ratio of IVIG.

Conclusion: SJS and TEN are two variants of the same spectrum, and they differ from each other not only in the severity of epidermal detachment but also in other clinical parameters and their distinct clinical courses. Thus, differential treatment of both conditions may have benefits for their prognosis.

Keywords: corticosteroids, intravenous immunoglobulin, Stevens-Johnson Syndrome, toxic epidermal necrolysis, cutaneous drug reaction

\section{Introduction}

Toxic epidermal necrolysis (TEN) and Stevens-Johnson Syndrome (SJS) are acute, potentially life-threatening skin and mucosal reactions, usually to drugs, which are characterized by epidermal detachment and mucositis. ${ }^{1}$ TEN occurs at an estimated incidence of $0.4-1.2$ cases per million people per year, ${ }^{2-5}$ with an appreciable mortality rate of $20 \%-30 \%$, which may be a conservative estimate given that TEN is underreported. ${ }^{6}$ For SJS, the incidence varies from one to six cases per million people per year, and the mortality rate is about 5\%.,5 The difference between SJS and TEN relates to how much of the body surface is affected: SJS consists of epidermal detachment of less than $10 \%$ of the body surface area; for TEN, epidermal detachment is more than $30 \%$ of the body surface; and for SJS/TEN overlap syndrome, epidermal detachment is between $10 \%$ and $30 \%{ }^{7}$ Histopathology is similar for both diseases, but varies in degree 
depending on severity of the condition. TEN is more severe than SJS with identical pathology. ${ }^{8}$ There is now consensus that SJS and TEN are variations of the same condition. ${ }^{7}$

No controlled trials of therapy for SJS or TEN have been documented to date. Systemic corticosteroids and immunosuppressive drugs are widely used in addition to supportive therapy to halt the progression of these diseases, which is based on the concept that they are T-cell-mediated diseases with $\mathrm{CD}^{+}$cells acting as the major mediator of keratinocyte death. ${ }^{9-11}$ It was reported that interactions between the death receptor Fas (CD95) and its ligand present on epidermal cells might play an important role in the apoptosis that characterizes TEN, so the use of intravenous immunoglobulin (IVIG) is often recommended. ${ }^{12}$

Thus far, controversy has existed in the literature in relation to the clinical definitions of these diseases and whether they are distinct entities or a spectrum of one disease process. For better understanding of the clinical characteristics and development of the two conditions, we performed this retrospective study to compare SJS and TEN in multi-aspect with regards to demographic information, clinical manifestations, and therapeutic responses.

\section{Methods}

We retrospectively reviewed the medical records of all patients admitted to the First Affiliated Hospital of Nanjing Medical University, Nanjing, the People's Republic of China, from January 2007 to December 2013 for SJS and TEN. For SJS, symptoms should include acute conditions characterized by mucous membrane erosions and skin lesions (described as macules, atypical target-like lesions, bulla, erosions) with less than $30 \%$ of maximum detachment of the skin surface area; for TEN, the symptoms should include more than $30 \%$ of maximum skin detachment in addition to the symptoms above. Based on the definition, SJS/TEN overlap cases were included in SJS. ${ }^{13-15}$ The case notes, charts, investigation results, and treatment records of these patients were retrospectively reviewed. Data obtained included the age, sex, medical history, presenting complaints, inciting drugs, duration between the initial consumption of the drug and the onset of symptoms, and score for toxic epidermal necrosis. ${ }^{16}$ Treatment regimens, the time from the initiation of therapy to control of the lesions (a halt of the progression of necrolysis and signs of re-epithelialization), ${ }^{17}$ duration of hospitalization, and mortality were also recorded. Since bicarbonate levels are not routinely determined in our department, that value was equally excluded when modified score for toxic epidermal necrosis (mSCORTEN) was calculated in the study.
Data analysis was performed using the Statistical Package for Social Science (SPSS for Windows, version 16.0). Data from the time of the initiation of therapy to control of the lesions, the maximum dosage of corticosteroid, and the duration of hospital stay were presented as mean \pm standard deviation, and the Kruskal-Wallis test for comparisons was performed. Categorical variables were compared among the groups of patients using the chi-square test. Multiple linear regression analysis was used to assess the relationship between the maximum dosage of corticosteroids and the year. We used binary logistic regression to study the relationship between the utilization rate of IVIG and the year, and the correlation between the albumin level and secondary infections. Cox regression analysis was used to analyze associations between the duration of the hospital stay and the maximum dosage of corticosteroids as well as the usage of IVIG, and also associations between the time from the initiation of therapy to control of the lesions and the maximum dosage of corticosteroids as well as the usage of IVIG. $P<0.05$ is considered statistically significant. All $P$-values are two-tailed.

\section{Results}

\section{Demographics and treatments}

Eighty-seven cases, including 39 SJS and 48 TEN, were studied. The age of SJS ranged from 7-84 years (average age, 44.15 \pm 19.45 years), and that of TEN ranged from 10-84 years (average age, 42.98 \pm 21.71 years). Females predominated in both the SJS and TEN, with a ratio of 1.17:1 (21:18) and 1.67:1 (30:18), respectively. Drugs considered to be related to SJS and TEN are listed in Table 1. These drugs were dominated by antiepileptics (38.4\%) and

Table I Demographics of the patients

\begin{tabular}{lll}
\hline Drug used & SJS (n) & TEN (n) \\
\hline Antiepileptics & 15 & 15 \\
Carbamazepine & 12 & 13 \\
Lamotrigine & 2 & 1 \\
Phenobarbital & 1 & 1 \\
Antibiotics & 9 & 6 \\
Amoxicillin & 3 & 1 \\
Cephalosporins & 4 & 3 \\
Metronidazole & 2 & 0 \\
Levofloxacin & 0 & 1 \\
Antipodagrics & 5 & 6 \\
$\quad$ Allopurinol & 5 & 6 \\
Nonsteroidal anti-inflammatory drugs & 1 & 10 \\
Traditional Chinese medicine & 3 & 6 \\
Other drugs & 6 & 5 \\
\hline Abbrevitions &
\end{tabular}

Abbreviations: $\mathrm{n}$, number; SJS, Stevens-Johnson Syndrome; TEN, toxic epidermal necrolysis. 
antibiotics (23.1\%) in SJS and by antiepileptics (31.3\%) and nonsteroidal anti-inflammatory drugs $(20.8 \%)$ in TEN. However, there was no significant difference in the constituent ratio of causative drugs between the two groups $(P=0.121)$.

The mean maximum doses of corticosteroids were $98.62 \pm 35.67 \mathrm{mg}$ in SJS and $190.87 \pm 131.41 \mathrm{mg}$ in TEN. The mean utilization rates of IVIG were $39.19 \%$ in SJS and $65.08 \%$ in TEN. The mean maximum dose of corticosteroids and the mean utilization rate of IVIG were higher in TEN than in $\operatorname{SJS}$ ( $P=0.000$ and $P=0.018$, respectively). However, the mean number of days of corticosteroids use along with maximum dose of corticosteroids was $6.38 \pm 3.02$ days in SJS and $6.13 \pm 3.58$ days in TEN, which was of no significant difference between SJS and TEN $(P=0.258)$.

\section{Comparisons of complications and mortality of SJS and TEN}

Hepatic involvement with elevation of liver enzymes (alanine transaminase $[\mathrm{ALT}]$ or aspartate transaminase $[\mathrm{AST}] \geq 2$ times the upper limit of normal), renal involvement with elevation of urea and creatinine (urea or creatinine was greater than the upper limit of normal) as well as hypoproteinemia and secondary infections are common complications in SJS and TEN. ${ }^{17}$ Abnormalities of ALT, AST, urea, and/or creatinine mostly occur in the first week after onset in TEN, while abnormalities of AST and urea usually occur in the first week after onset in SJS. However, abnormalities of ALT mostly occur in the second week after onset in SJS, and there was no abnormality of creatinine in SJS (Figure 1A and B). Hypoproteinemia mostly occurred in SJS and TEN patients in the second week after onset (Figure 1C). There was no difference in the incidence of hepatic involvement and renal involvement between SJS and TEN $(P=0.138$ and $P=0.210$, respectively). However, the incidence rate of hypoproteinemia and secondary infections is significantly higher in TEN than in SJS ( $P=0.001$ and $P=0.002$, respectively). Two cases died of TEN $(4.2 \%, 2: 48)$, while no patient died in the SJS group. No significant difference was found in mortality ratio between the two groups ( $P=0.502$, according to Fisher exact test).
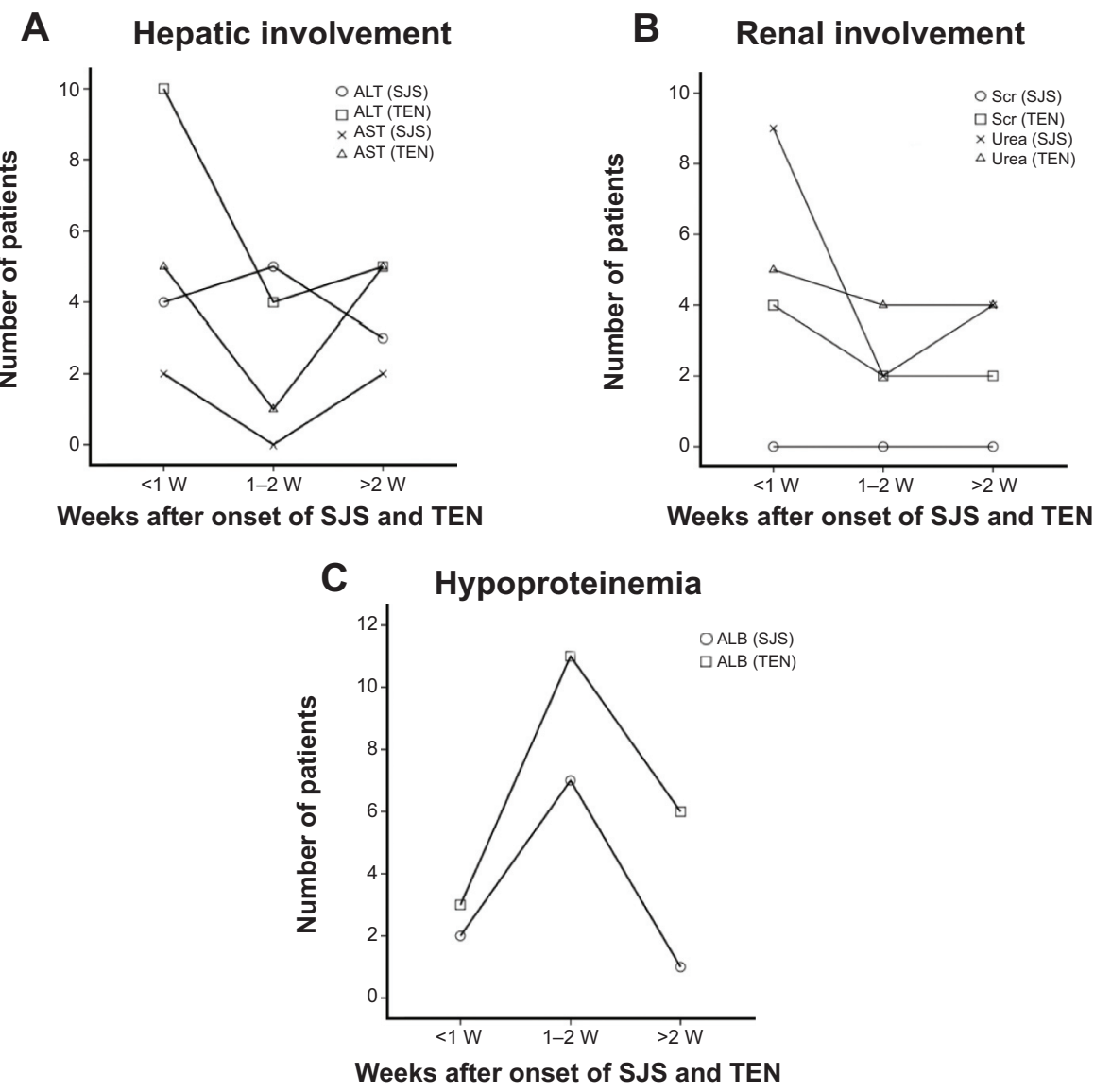

Figure I Hepatic involvement (A), renal involvement (B), and hypoproteinemia (C) distribution in patients with TEN and SJS.

Abbreviations: ALB, albumin; ALT, alanine transaminase; AST, aspartate transaminase; Scr, serum creatinine; SJS, Stevens-Johnson Syndrome; TEN, toxic epidermal necrolysis; W, weeks. 
Table 2 Clinical characteristics of SJS and TEN grouped according to albumin level quartile values used as cutoff points

\begin{tabular}{|c|c|c|c|c|c|c|c|}
\hline \multirow[t]{2}{*}{ Variable } & \multirow[t]{2}{*}{ Group } & \multicolumn{4}{|l|}{ Albumin levels } & \multirow[t]{2}{*}{ F or $\chi^{2}$} & \multirow[t]{2}{*}{$P$-value } \\
\hline & & $0-25.8 \mathrm{~g} / \mathrm{L}$ & $25.9-31 \mathrm{~g} / \mathrm{L}$ & $31.1-35.1 \mathrm{~g} / \mathrm{L}$ & $\geq 35.1 \mathrm{~g} / \mathrm{L}$ & & \\
\hline \multirow[t]{2}{*}{ Duration of hospital stay (days) } & SJS & $18.62 \pm 5.44$ & $15.29 \pm 3.99$ & $12.14 \pm 2.34$ & $|4.50 \pm 3.4|$ & 8.410 & 0.380 \\
\hline & TEN & $25.00 \pm 10.43$ & $22.00 \pm 9.53$ & $19.08 \pm 7.16$ & $18.55 \pm 7.66$ & 2.080 & 0.550 \\
\hline Time from initiation of therapy & SJS & $3.50 \pm 2.24$ & $3.00 \pm 1.63$ & $4.00 \pm 2.20$ & $3.40 \pm 1.43$ & 0.902 & 0.825 \\
\hline to control of lesions (days) & TEN & $5.50 \pm 3.12$ & $5.12 \pm 3.10$ & $5.69 \pm 2.59$ & $4.82 \pm 2.56$ & 0.785 & 0.853 \\
\hline \multirow[t]{2}{*}{ Maximum dosage of steroid (mg) } & SJS & $94.29 \pm 25.33$ & $85.7 \mid \pm 15.12$ & $87.50 \pm 21.21$ & $122.60 \pm 55.10$ & 5.069 & 0.167 \\
\hline & TEN & $212.50 \pm 129.13$ & $185.50 \pm 120.69$ & $|75.08 \pm| 26.4 \mid$ & $191.82 \pm 163.39$ & 1.063 & 0.786 \\
\hline \multirow[t]{2}{*}{ Number of usages of IVIG } & SJS & 12 & 2 & 2 & I & 16.322 & 0.000 \\
\hline & TEN & 9 & 10 & 10 & 4 & 6.849 & 0.095 \\
\hline
\end{tabular}

Note: Data shown are mean \pm standard deviation.

Abbreviations: IVIG, intravenous immunoglobin; SJS, Stevens-Johnson Syndrome; TEN, toxic epidermal necrolysis.

\section{Hypoproteinemia}

Table 2 shows the clinical characteristics of SJS and TEN grouped according to albumin levels, quartile values of which were used as cutoff points. Patients in quartiles I, II, III, and IV had increasingly higher albumin levels. Among SJS patients, the distribution of the duration of hospital stays $(P=0.38)$, the time from the initiation of therapy to control of the lesions $(P=0.825)$ and the maximum dosage of corticosteroids $(P=0.167)$ were similar among the four groups. In contrast, the frequency distribution of the use of IVIG $(P=0.00)$ differed among the groups; however, among TEN patients, all were similar among the four groups. The results of the binary logistic regression analysis indicated that albumin levels are significantly associated with secondary infections among TEN ( $\beta=-0.201, P=0.009$ ) and are not significantly associated with secondary infections among SJS $(\beta=-0.019, P=0.808)$.

\section{Comparison of corticosteroids usage in succeeding years}

To examine the independent association between the treatment year and the maximum dosage of corticosteroids, multiple linear regression analysis was performed. In this model, the maxi- mum dosage of corticosteroids was employed as the dependent variable, and independent variables included the year, age, sex, and mSCORTEN. In the final model, among SJS and TEN patients, sex $\left(\beta_{\mathrm{SJS}}=-0.029, P_{\mathrm{SJS}}=0.863 ; \beta_{\mathrm{TEN}}=0.104\right.$, $P_{\mathrm{TEN}}=0.496$, respectively), age $\left(\beta_{\mathrm{SJS}}=0.060, P_{\mathrm{SJS}}=0.710\right.$; $\beta_{\mathrm{TEN}}=0.115, P_{\mathrm{TEN}}=0.513$, respectively), and mSCORTEN $\left(\beta_{\mathrm{SJS}}=0.000, P_{\mathrm{SJS}}=0.998 ; \beta_{\mathrm{TEN}}=-0.052, P_{\mathrm{TEN}}=0.766\right.$, respectively) have no effect on the maximum dosage of corticosteroids, while each successive year of treatment $\left(\beta_{\mathrm{SJS}}=-0.524\right.$, $P_{\mathrm{SJS}}=0.002 ; \beta_{\mathrm{TEN}}=-0.461, P_{\mathrm{TEN}}=0.004$, respectively) leads to a gradual decrease to it in both diseases. However, the severity of both diseases was the same, since there was no significant difference in mSCORTEN during these years (Figure 2).

\section{Comparison of IVIG usage in succeeding years}

To evaluate the association between the utilization rate of IVIG and the treatment year, binary logistic regression analysis was conducted. In the analysis, the utilization rate of IVIG was employed as the dependent variable, while the covariate variables included age, sex, mSCORTEN, and the year. The results of that analysis (Table 3 ) indicated that the treatment year was significantly associated with the utilization rate of
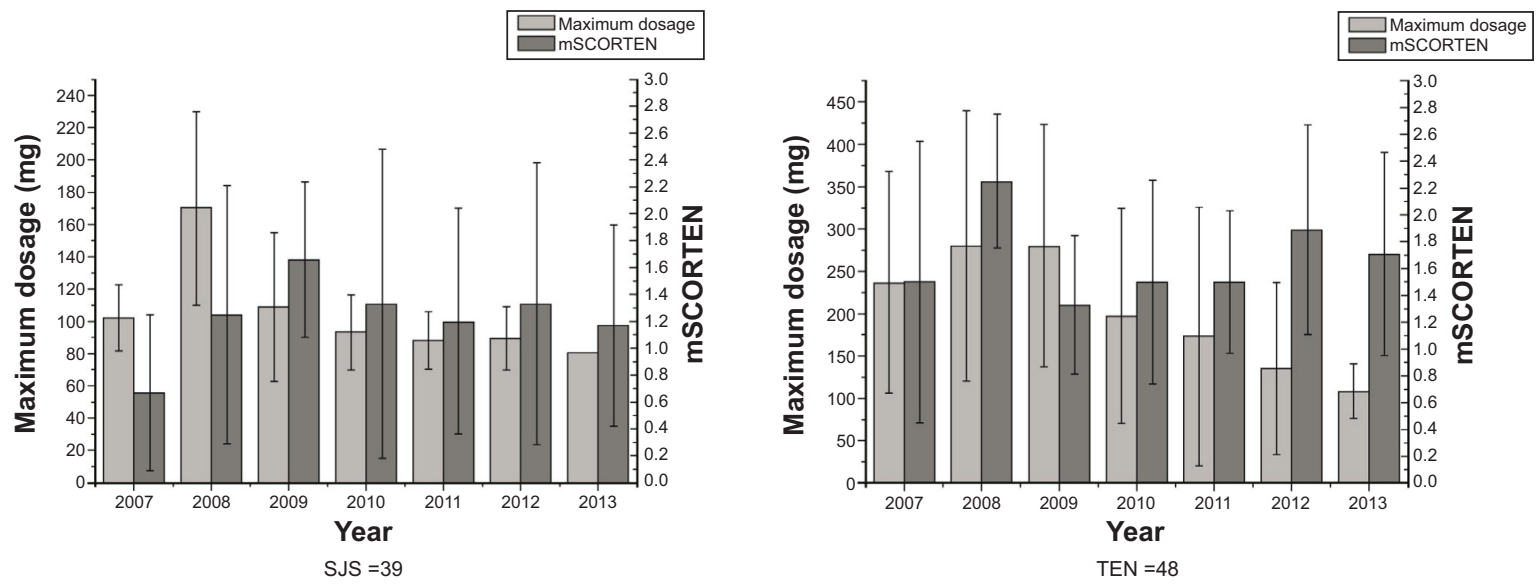

Figure 2 Corticosteroid usage and mSCORTEN in succeeding years in SJS patients and TEN patients.

Abbreviations: mSCORTEN, modified score for toxic epidermal necrosis; SJS, Stevens-Johnson Syndrome; TEN, toxic epidermal necrolysis. 
Table 3 Results of the binary logistic regression analysis

\begin{tabular}{llll}
\hline Effect & Group & $\beta$ & $P$-value \\
\hline Constant & SJS & $-1.179 E 3$ & 0.038 \\
& TEN & -495.446 & 0.198 \\
Sex & SJS & 0.346 & 0.678 \\
& TEN & 0.648 & 0.393 \\
Age & SJS & -0.024 & 0.250 \\
& TEN & -0.028 & 0.150 \\
mSCORTEN & SJS & 1.117 & 0.037 \\
\multirow{2}{*}{ Year } & TEN & 0.050 & 0.914 \\
& SJS & 0.586 & 0.038 \\
& TEN & 0.247 & 0.198 \\
\hline
\end{tabular}

Abbreviations: mSCORTEN, modified score for toxic epidermal necrosis; SJS, Stevens-Johnson Syndrome; TEN, toxic epidermal necrolysis.

IVIG among SJS patients ( $\beta=1.796, P=0.038)$, but there was no significant association with the utilization rate of IVIG among TEN patients $(\beta=1.280, P=0.198)$. Therefore, the number of SJS patients who used IVIG increased in succeeding years, while the number of TEN patients who used IVIG was constant over this period (Figure 3).

\section{Associations between the duration of the hospital stay and the maximum dosage of corticosteroids as well as the usage of IVIG}

To evaluate the associations between the duration of the hospital stay and the maximum dosage of corticosteroids as well as the usage of IVIG, Cox regression analysis was conducted. In the analysis, the duration of the hospital stay



Figure 3 IVIG usage in succeeding years in SJS patients and TEN patients. Abbreviations: IVIG, intravenous immunoglobin; SJS, Stevens-Johnson Syndrome; TEN, toxic epidermal necrolysis. was employed as the dependent variable, while the covariate variables included age, sex, mSCORTEN, the maximum dosage of corticosteroids, and the usage of IVIG. The results of the Cox regression analysis (Table 4) indicated that the duration of the hospital stay was significantly associated with the usage of IVIG $\left(\beta_{\text {SJS }}=-0.922, P_{\text {SJS }}=0.030 ; \beta_{\text {TEN }}=-1.046\right.$, $\left.P_{\text {TEN }}=0.016\right)$, but there was no significant association with the maximum dosage of corticosteroids $\left(\beta_{S J S}=-0.006\right.$, $\left.P_{\text {SJS }}=0.202 ; \beta_{\text {TEN }}=-0.003, P_{\text {TEN }}=0.063\right)$ among SJS and TEN patients.

Associations between the time from the initiation of therapy to control of the lesions and the maximum dosage of corticosteroids as well as the usage of IVIG

To evaluate the associations between the time from initiation of therapy to control of the lesions and the maximum dosage of corticosteroids as well as the usage of IVIG, Cox regression analysis was conducted. In that analysis, the time from the initiation of therapy to the control of lesions was employed as the dependent variable, while the covariate variables included age, sex, mSCORTEN, the maximum dosage of corticosteroids, and the usage of IVIG. The results of the Cox regression analysis (Table 5) indicated that the time from the initiation of therapy to control of the lesions was significantly associated with the usage of IVIG as well as the maximum dosage of corticosteroids among TEN patients, $(\beta$ corticosteroids $=-0.003$, Pcorticosteroids $\left.=0.028 ; \beta_{I V I G}=-0.71, P_{I V I G}=0.046\right)$. However, there was no significant association with the usage of IVIG as well as the maximum dosage of corticosteroids ( $\beta$ corticosteroids $=0.001$, Pcorticosteroids $=0.81 ; \beta_{I V I G}=0.512$, $\left.P_{I V I G}=0.19\right)$ among SJS patients.

Table 4 Results of the Cox regression analysis of associations between duration of hospital stay and the maximum dosage of corticosteroids as well as the usage of IVIG

\begin{tabular}{llll}
\hline Variable & Group & $\boldsymbol{\beta}$ & $\boldsymbol{P}$-value \\
\hline Sex & SJS & -0.568 & 0.127 \\
& TEN & -0.289 & 0.442 \\
Age & SJS & 0.013 & 0.215 \\
& TEN & 0.001 & 0.905 \\
mSCORTEN & SJS & -0.336 & 0.219 \\
& TEN & 0.106 & 0.623 \\
Maximum dosage & SJS & -0.006 & 0.202 \\
of corticosteroids & TEN & -0.003 & 0.063 \\
Usage of IVIG & SJS & -0.992 & 0.030 \\
& TEN & -1.046 & 0.016 \\
\hline
\end{tabular}

Abbreviations: IVIG, intravenous immunoglobin; mSCORTEN, modified score for toxic epidermal necrosis; SJS, Stevens-Johnson Syndrome; TEN, toxic epidermal necrolysis. 
Table 5 Results of the Cox regression analysis of associations between time from initiation of therapy to control of the lesions and the maximum dosage of corticosteroids as well as the usage of IVIG

\begin{tabular}{llll}
\hline Variable & Group & $\boldsymbol{\beta}$ & $\boldsymbol{P}$-value \\
\hline Sex & SJS & -0.668 & 0.076 \\
& TEN & 0.441 & 0.183 \\
Age & SJS & 0.011 & 0.286 \\
& TEN & -0.011 & 0.309 \\
mSCORTEN & SJS & -0.065 & 0.786 \\
& TEN & -0.133 & 0.601 \\
Maximum dosage & SJS & 0.001 & 0.810 \\
of corticosteroids & TEN & -0.003 & 0.028 \\
Usage of IVIG & SJS & 0.512 & 0.190 \\
& TEN & -0.710 & 0.046 \\
\hline
\end{tabular}

Abbreviations: IVIG, intravenous immunoglobin; mSCORTEN, modified score for toxic epidermal necrosis; SJS, Stevens-Johnson Syndrome; TEN, toxic epidermal necrolysis.

\section{Discussion}

TEN and SJS are rare, potentially life-threatening acute exfoliative drug-induced skin and mucosal disorders. ${ }^{18}$ The categorization of erythema multiforme (EM), SJS, and TEN remains a topic of ongoing controversy because of the incomplete elucidation of their pathogenesis. The first description of TEN in 1956 by Lyell ${ }^{19}$ made no reference to EM or SJS. However, it soon became evident that severe forms of SJS could evolve to TEN and that the same drugs could induce both disorders. Because detachment of the epidermis is a major prognosis factor, Bastuji-Garin et $\mathrm{al}^{20}$ proposed SJS, SJS/TEN overlap, and TEN as classifications based on the severity of the epidermal detachment. Epidermal detachment of less than $10 \%$ of the body surface area is classified as SJS, more than $30 \%$ as TEN, and $10 \%-30 \%$ as SJS/TEN overlap..$^{21,22}$ The present opinion is that SJS and TEN are severity variants of the same drug-induced process. However, until it has been clarified that common etiologies and pathologic process are involved, some experts believe that this classification scheme is imperfect. ${ }^{7,23,24}$

Our study is the first to compare SJS and TEN from multiple aspects such as clinical features and therapeutic responses. In contrast to earlier studies ${ }^{25}$ showing that the incidences of hepatic involvement and renal involvement in TEN are higher than in SJS, our series had an equal incidence. Abnormalities of ALT, AST, urea, and creatinine in TEN usually occur within the first week after the onset, while abnormalities of AST and urea usually occur in SJS within the first week after onset. However, the abnormality of ALT in SJS usually occurs within the second week after the onset, and there is no abnormality of creatinine in SJS.
Although hepatic and renal involvement in TEN and SJS usually occur within the first week after the onset of the diseases, it should be noted that delayed hepatic and renal involvement will occur in a considerable number of patients. Therefore, clinicians should closely monitor liver and kidney functions in later stages of the diseases.

In the course of SJS and TEN, hypoproteinemia ought to be considered as a solitary complication regarding its strategic role in the prognosis of the disease and its response to drug therapy. Not only can hypoproteinemia induce an increased incidence of infections and slow the healing of skin lesions, but it also decreases the therapeutic effect of certain drugs because albumin can be used as a carrier for many drugs such as corticosteroids and antibiotics. The incidence rates of hypoproteinemia and secondary infections are significantly higher in TEN than in SJS. Hypoproteinemia mostly occurs in SJS and TEN in the second week after the onset, which is mainly due to the loss of plasma after the outbreak of vesiculobullous. In this study, the incidence of secondary infections increases with the decrease in serum albumin levels in TEN, while there is no significant association between secondary infections and serum albumin levels in SJS using the binary logistic regression analysis. The lower the albumin levels are, the higher the incidences of infection and edema are, and the slower the onset time of corticosteroids therapy is. The disease conditions of patients whose albumin levels are lower are usually worse, which might make doctors choose IVIG as a supplementary therapy. In our study, the IVIG differed significantly among the four groups of various albumin levels in SJS patients.

There is currently no specific or classified treatment for TEN or SJS because of their complex pathogenesis. Systemic administration of corticosteroids and IVIG are still controversial in many countries, but these two treatments have become mainstream in the People's Republic of China.

The use of corticosteroids is based on the idea that corticosteroids probably suppress immunological responses by inhibiting the functions of cytotoxic T lymphocytes and also inhibit interferon gamma mediated apoptosis. ${ }^{26}$ Moreover, corticosteroids have pleomorphic effects on the immune system, including the inhibition of chemokines and numerous cytokines like TNF-alpha. ${ }^{27}$ In 1983, Kim et al ${ }^{28}$ suggested that treatment of SJS and TEN with glucocorticoids is associated with increased morbidity and mortality mainly due to secondary infections. Since the 1980s, several studies stated that corticosteroids not only enhance the risk of sepsis but also delay the epithelialization. ${ }^{29,30}$ However, In a case-control analysis of patients selected from EuroSCAR/RegiSCAR 
studies, it was shown that long-term corticosteroid use prior to the onset of disease may prolong the latency (time from drug initiation to the onset of disease) and progression of disease but does not influence severity or mortality. ${ }^{31}$ In this study, we concluded that the maximum dosage of corticosteroids gradually decreased with the increase of the year in the two diseases using multiple linear regression analysis. Nevertheless, the severities of the diseases are the same according to the insignificant difference in mSCORTEN during these years. The maximum dosage of corticosteroids also has no influence on the time from the initiation of therapy to the control of the lesions in SJS. With an increase in the maximum dosage of corticosteroids, the time from the initiation of therapy to the control of the lesions becomes shorter and shorter in TEN. Therefore, we conclude that there is a difference in the response to corticosteroid therapy between SJS and TEN. The maximum dosage of corticosteroids decreasing with years of treatment might be ascribed to the fact that, to some extent, Chinese dermatologists are much more aware of the update of the strategic and double-sided role of corticosteroids, along with controllable and different dose ranges in treatment of SJS and TEN. SJS and TEN belong to severe drug eruption and are often accompanied by systemic involvement; after all, they manifest transient disease process. Most of the patients would be cured by treatment at last. The death or complications of a considerable number of patients occurring later in the disease may be caused by excessive use of corticosteroids. The dermatologists should weigh benefits and side effects of certain doses of corticosteroids in the treatment of SJS and TEN. In practice, it is relatively difficult to determine the precise doses of corticosteroids for the particular conditions. In our opinion, the doses of corticosteroids that can control temperature and the rapid development of lesions in the case of supportive treatment should be considered adequate. The diseases can be controlled gradually.

IVIG seems to have many advantages in the treatment of TEN and SJS. In 1998, Viard et al ${ }^{12}$ published a new proposal for therapy based on the use of IVIG, with excellent clinical findings, on the basis of their ability to inhibit the Fas-Fas ligand-mediated apoptosis of keratinocytes. However, a prospective noncomparative study performed by Bachot et $\mathrm{a}^{132}$ and a single institutional analysis with nonimmunoglobin-treated controls carried out by Brown et $\mathrm{a}^{33}$ do not show a significant improvement in the mortality of TEN patients treated with IVIG and may indicate a potential detriment in their use, especially in elderly patients and in patients with impaired renal function. On the contrary,
Metry et al,${ }^{34}$ Tristani-Firouzi et al, ${ }^{35}$ Aires et al ${ }^{36}$ and Trent et $\mathrm{al}^{37}$ conducted a number of retrospective and prospective studies to examine the efficacy and safety of IVIG in TEN patients and found that IVIG has a beneficial effect in TEN. The total IVIG therapeutic dose was also set at $2 \mathrm{~g} / \mathrm{kg}$ in five infusions in the present study, which is consistent with empirical therapeutic dose reported in the literatures. With an increasing utilization ratio of IVIG, the length of the hospital stay has become significantly shorter, whereas the time from the initiation of therapy to control of the lesions remains the same in SJS. For TEN, both the length of the hospital stay and the time from the initiation of therapy to control of the lesions has become shorter as an increasing utilization ratio of IVIG. Therefore, we deduced that there is also a difference in the response to IVIG between SJS and TEN.

Earlier studies had shown that there might be different responses to corticosteroids and IVIG between SJS and TEN. Tripathi et al ${ }^{38}$ concluded that SJS must be distinguished from TEN since SJS is steroid responsive, but TEN has not been observed to improve with systemic corticosteroids. Tan et $\mathrm{al}^{39}$ had inferred from a small sample (only three cases of TEN, seven cases of SJS/TEN overlap, and 18 cases of SJS) and a descriptive survey without a strict statistical analysis that steroid therapy with high-dose corticosteroids was effective in SJS, whereas IVIG showed efficacy in TEN according to their experience. In any case, there is no such comparative study using the regression model which simultaneously balances and excludes other possible impacting factors focusing on evaluation of the efficacy of IVIG in the treatment of TEN and SJS in the published literature. The results we obtained from this study indicate that corticosteroids and IVIG are effective in shortening the time from the initiation of therapy to control of the lesions in TEN while both corticosteroids and IVIG are not effective in shortening the time from the initiation of therapy to the control of lesions in SJS, which confirms our previous presumption and allows a better understanding of different responses to corticosteroids and IVIG of SJS and TEN.

SJS varies from TEN in clinical manifestations, therapeutic responses to corticosteroids, and IVIG, probably due to distinguished pathogenesis of both diseases, which is not covered in this study. Nevertheless, the variation could not be totally attributed to differences of severity related to the extent of diseases. We consider SJS and TEN as two variants of the same spectrum, and they differ from each other not only in the severity of epidermal detachment but also in other clinical parameters and their distinct clinical courses. 
Thus, we believe that differential treatment of both conditions may have benefits for their prognosis.

\section{Acknowledgment}

This work was supported by the National Natural Science Foundation of China (Grant number 81171517).

\section{Disclosure}

The authors report no conflicts of interest in this work.

\section{References}

1. Lee HY, Chung WH. Toxic epidermal necrolysis: the year in review. Curr Opin Allergy Clin Immunol. 2013;13(4):330-336.

2. Naveen KN, Pai VV, Rai V, Athanikar SB. Retrospective analysis of Steven Johnson syndrome and toxic epidermal necrolysis over a period of 5 years from northern Karnataka, India. Indian J Pharmacol. 2013;45(1):80-82.

3. Downey A, Jackson C, Harun N, Cooper A. Toxic epidermal necrolysis: review of pathogenesis and management. J Am Acad Dermatol. 2012; 66(6):995-1003.

4. Gerull R, Nelle M, Schaible T. Toxic epidermal necrolysis and Stevens-Johnson syndrome: a review. Crit Care Med. 2011;39(6): $1521-1532$

5. Chan HL, Stern RS, Arndt KA, et al. The incidence of erythema multiforme, Stevens-Johnson syndrome, and toxic epidermal necrolysis. A population-based study with particular reference to reactions caused by drugs among outpatients. Arch Dermatol. 1990;126(1):43-47.

6. Mittmann N, Knowles SR, Gomez M, Fish JS, Cartotto R, Shear NH. Evaluation of the extent of under-reporting of serious adverse drug reactions: the case of toxic epidermal necrolysis. Drug Saf. 2004;27(7):477-487.

7. Schwartz RA, McDonough PH, Lee BW. Toxic epidermal necrolysis: Part I. Introduction, history, classification, clinical features, systemic manifestations, etiology, and immunopathogenesis. J Am Acad Dermatol. 2013;69(2):173.e1-e13; quiz 185.

8. Lissia M, Mulas P, Bulla A, Rubino C. Toxic epidermal necrolysis (Lyell's disease). Burns. 2010;36(2):152-163.

9. Nassif A, Bensussan A, Boumsell L, et al. Toxic epidermal necrolysis: effector cells are drug-specific cytotoxic T cells. J Allergy Clin Immunol. 2004;114(5):1209-1215.

10. Nassif A, Bensussan A, Dorothée G, et al. Drug specific cytotoxic T-cells in the skin lesions of a patient with toxic epidermal necrolysis. J Invest Dermatol. 2002;118(4):728-733.

11. Paquet P, Piérard GE, Quatresooz P. Novel treatments for drug-induced toxic epidermal necrolysis (Lyell's syndrome). Int Arch Allergy Immunol. 2005;136(3):205-216.

12. Viard I, Wehrli P, Bullani R, et al. Inhibition of toxic epidermal necrolysis by blockade of CD95 with human intravenous immunoglobulin. Science. 1998;282(5388):490-493.

13. Yamane Y, Aihara M, Ikezawa Z. Analysis of Stevens-Johnson syndrome and toxic epidermal necrolysis in Japan from 2000 to 2006. Allergol Int. 2007;56(4):419-425.

14. Chen J, Wang B, Zeng Y, Xu H. High-dose intravenous immunoglobulins in the treatment of Stevens-Johnson syndrome and toxic epidermal necrolysis in Chinese patients: a retrospective study of 82 cases. Eur J Dermatol. 2010;20(6):743-747.

15. Kamaliah MD, Zainal D, Mokhtar N, Nazmi N. Erythema multiforme, Stevens-Johnson syndrome and toxic epidermal necrolysis in northeastern Malaysia. Int J Dermatol. 1998;37(7):520-523.

16. Bastuji-Garin S, Fouchard N, Bertocchi M, Roujeau JC, Revuz J, Wolkenstein P. SCORTEN: a severity-of-illness score for toxic epidermal necrolysis. J Invest Dermatol. 2000;115(2):149-153.
17. Rajaratnam R, Mann C, Balasubramaniam P, et al. Toxic epidermal necrolysis: retrospective analysis of 21 consecutive cases managed at a tertiary centre. Clin Exp Dermatol. 2010;35(8):853-862.

18. Barvaliya M, Sanmukhani J, Patel T, Paliwal N, Shah H, Tripathi C. Drug-induced Stevens-Johnson syndrome (SJS), toxic epidermal necrolysis (TEN), and SJS-TEN overlap: a multicentric retrospective study. J Postgrad Med. 2011;57(2):115-119.

19. Lyell A. Toxic epidermal necrolysis: an eruption resembling scalding of the skin. Br J Dermatol. 1956;68(11):355-361.

20. Bastuji-Garin S, Rzany B, Stern RS, Shear NH, Naldi L, Roujeau JC. Clinical classification of cases of toxic epidermal necrolysis, StevensJohnson syndrome, and erythema multiforme. Arch Dermatol. 1993;129(1):92-96.

21. Sharma VK, Sethuraman G. Adverse cutaneous reactions to drugs: an overview. J Postgrad Med. 1996;42(1):15-22.

22. Mockenhaupt M. Severe drug-induced skin reactions: clinical pattern, diagnostics and therapy. J Dtsch Dermatol Ges. 2009;7(2):142-60; quiz 161.

23. Wolf R, Lipozencic J. Shape and configuration of skin lesions: targetoid lesions. Clin Dermatol. 2011;29(5):504-508.

24. Roujeau JC. The spectrum of Stevens-Johnson syndrome and toxic epidermal necrolysis: a clinical classification. J Invest Dermatol. 1994;102(6):28S-30S.

25. Thammakumpee J, Yongsiri S. Characteristics of toxic epidermal necrolysis and Stevens-Johnson syndrome: a 5-year retrospective study. J Med Assoc Thai. 2013;96(4):399-406.

26. Jagadeesan S, Sobhanakumari K, Sadanandan SM, et al. Low dose intravenous immunoglobulins and steroids in toxic epidermal necrolysis: a prospective comparative open-labelled study of 36 cases. Indian J Dermatol Venereol Leprol. 2013;79(4):506-511.

27. Kardaun SH, Jonkman MF. Dexamethasone pulse therapy for StevensJohnson syndrome/toxic epidermal necrolysis. Acta Derm Venereol. 2007; 87(2):144-148.

28. Kim PS, Goldfarb IW, Gaisford JC, Slater H. Stevens-Johnson syndrome and toxic epidermal necrolysis: a pathophysiologic review with recommendations for a treatment protocol. J Burn Care Rehabil. 1983;4(2):91-100.

29. Halebian PH, Corder VJ, Madden MR, Finklestein JL, Shires GT. Improved burn center survival of patients with toxic epidermal necrolysis managed without corticosteroids. Ann Surg. 1986; 204(5):503-512.

30. Murphy JT, Purdue GF, Hunt JL. Toxic epidermal necrolysis. J Burn Care Rehabil. 1997;18(5):417-420.

31. Lee HY, Dunant A, Sekula P, et al. The role of prior corticosteroid use on the clinical course of Stevens-Johnson syndrome and toxic epidermal necrolysis: a case-control analysis of patients selected from the multinational EuroSCAR and RegiSCAR studies. Br J Dermatol. 2012;167(3):555-562.

32. Bachot N, Revuz J, Roujeau JC. Intravenous immunoglobulin treatment for Stevens-Johnson syndrome and toxic epidermal necrolysis: a prospective noncomparative study showing no benefit on mortality or progression. Arch Dermatol. 2003;139(1):33-36.

33. Brown KM, Silver GM, Halerz M, Walaszek P, Sandroni A, Gamelli RL. Toxic epidermal necrolysis: does immunoglobulin make a difference? J Burn Care Rehabil. 2004;25(1):81-88.

34. Metry DW, Jung P, Levy ML. Use of intravenous immunoglobulin in children with stevens-johnson syndrome and toxic epidermal necrolysis: seven cases and review of the literature. Pediatrics. 2003;112(6 Pt 1): $1430-1436$.

35. Tristani-Firouzi P, Petersen MJ, Saffle JR, Morris SE, Zone JJ. Treatment of toxic epidermal necrolysis with intravenous immunoglobulin in children. J Am Acad Dermatol. 2002;47(4):548-552.

36. Aires DJ, Fraga G, Korentager R, et al. Early treatment with nonsucrose intravenous immunoglobulin in a burn unit reduces toxic epidermal necrolysis mortality. J Drugs Dermatol. 2013;12(6): 679-684. 
37. Trent JT, Kirsner RS, Romanelli P, Kerdel FA. Analysis of intravenous immunoglobulin for the treatment of toxic epidermal necrolysis using SCORTEN: The University of Miami Experience. Arch Dermatol. 2003;139(1):39-43.

38. Tripathi A, Ditto AM, Grammer LC, et al. Corticosteroid therapy in an additional 13 cases of Stevens-Johnson syndrome: a total series of 67 cases. Allergy Asthma Proc. 2000;21(2):101-105.
39. Tan SK, Tay YK. Profile and pattern of Stevens-Johnson syndrome and toxic epidermal necrolysis in a general hospital in Singapore: treatment outcomes. Acta Derm Venereol. 2012;92(1):62-66.

\section{Publish your work in this journal}

Drug Design, Development and Therapy is an international, peerreviewed open-access journal that spans the spectrum of drug design and development through to clinical applications. Clinical outcomes, patient safety, and programs for the development and effective, safe, and sustained use of medicines are a feature of the journal, which has also been accepted for indexing on PubMed Central. The manuscript management system is completely online and includes a very quick and fair peer-review system, which is all easy to use. Visit http://www.dovepress.com/testimonials.php to read real quotes from published authors.

Submit your manuscript here: http://www.dovepress.com/drug-design-development-and-therapy-journal 